\title{
MUTT: Metric Unit TesTing for Language Generation Tasks
}

\author{
Willie Boag, Renan Campos, Kate Saenko, Anna Rumshisky \\ Dept. of Computer Science \\ University of Massachusetts Lowell \\ 198 Riverside St, Lowell, MA 01854 \\ \{wboag, rcampos, saenko, arum\}@cs.uml. edu
}

\begin{abstract}
Precise evaluation metrics are important for assessing progress in high-level language generation tasks such as machine translation or image captioning. Historically, these metrics have been evaluated using correlation with human judgment. However, human-derived scores are often alarmingly inconsistent and are also limited in their ability to identify precise areas of weakness. In this paper, we perform a case study for metric evaluation by measuring the effect that systematic sentence transformations (e.g. active to passive voice) have on the automatic metric scores. These sentence "corruptions" serve as unit tests for precisely measuring the strengths and weaknesses of a given metric. We find that not only are human annotations heavily inconsistent in this study, but that the Metric Unit TesT analysis is able to capture precise shortcomings of particular metrics (e.g. comparing passive and active sentences) better than a simple correlation with human judgment can.
\end{abstract}

\section{Introduction}

The success of high-level language generation tasks such as machine translation (MT), paraphrasing and image/video captioning depends on the existence of reliable and precise automatic evaluation metrics.

\begin{tabular}{|lll|}
\hline A man is holding a frog & There is no man holding a frog & 2.1 \\
The man is playing with a skull & There is no man playing with a skull & 2.3 \\
A man is driving a car & There is no man driving the car & 3.6 \\
A woman is combing her hair & There is no woman combing her hair & 3.6 \\
A man is walking outside & There is no man walking outside & 4.6 \\
A man is playing soccer & There is no man playing soccer & 4.8 \\
\end{tabular}

Figure 1: A few select entries from the SICK dataset. All of these entries follow the same "Negated Subject" transformation between sentence 1 and sentence 2 , yet humans annotated them with an inconsistently wide range of scores (from 1 to 5). Regardless of whether the gold labels for this particular transformation should score this high or low, they should score be scored consistently.

Efforts have been made to create standard metrics (Papineni et al., 2001; Lin, 2004; Denkowski and Lavie, 2014; Vedantam et al., 2014) to help advance the state-of-the-art. However, most such popular metrics, despite their wide use, have serious deficiencies. Many rely on ngram matching and assume that annotators generate all reasonable reference sentences, which is infeasible for many tasks. Furthermore, metrics designed for one task, e.g., MT, can be a poor fit for other tasks, e.g., video captioning.

To design better metrics, we need a principled approach to evaluating their performance. Historically, MT metrics have been evaluated by how well they correlate with human annotations (CallisonBurch et al., 2010; Machacek and Bojar, 2014). However, as we demonstrate in Sec. 5, human judgment can result in inconsistent scoring. This presents a serious problem for determining whether 
a metric is "good" based on correlation with inconsistent human scores. When "gold" target data is unreliable, even good metrics can appear to be inaccurate.

Furthermore, correlation of system output with human-derived scores typically provides an overall score but fails to isolate specific errors that metrics tend to miss. This makes it difficult to discover system-specific weaknesses to improve their performance. For instance, an ngram-based metric might effectively detect non-fluent, syntactic errors, but could also be fooled by legitimate paraphrases whose ngrams simply did not appear in the training set. Although there has been some recent work on paraphrasing that provided detailed error analysis of system outputs (Socher et al., 2011; Madnani et al., 2012), more often than not such investigations are seen as above-and-beyond when assessing metrics.

The goal of this paper is to propose a process for consistent and informative automated analysis of evaluation metrics. This method is demonstrably more consistent and interpretable than correlation with human annotations. In addition, we extend the SICK dataset to include un-scored fluency-focused sentence comparisons and we propose a toy metric for evaluation.

The rest of the paper is as follows: Section 2 introduces the corruption-based metric unit testing process, Section 3 lists the existing metrics we use in our experiments as well as the toy metric we propose, Section 4 describes the SICK dataset we used for our experiments, Section 5 motivates the need for corruption-based evaluation instead of correlation with human judgment, Section 6 describes the experimental procedure for analyzing the metric unit tests, Section 7 analyzes the results of our experiments, and in Section 8 we offer concluding remarks.

\section{Metric Unit TesTs}

We introduce metric unit tests based on sentence corruptions as a new method for automatically evaluating metrics developed for language generation tasks. Instead of obtaining human ranking for system output and comparing it with the metricbased ranking, the idea is to modify existing ref- erences with specific transformations, and examine the scores assigned by various metrics to such corruptions. In this paper, we analyze three broad categories of transformations - meaning-altering, meaning-preserving, and fluency-disrupting sentence corruptions - and we evaluate how successfully several common metrics can detect them.

As an example, the original sentence "A man is playing a guitar." can be corrupted as follows:

Meaning-Altering: A man is not playing guitar. Meaning-Preserving: A guitar is being played by a man.

Fluency-Disrupting: A man a guitar is playing.

Examples for each corruption type we consider are shown in Tables 1 and 2 .

\subsection{Meaning-altering corruptions}

Meaning-altering corruptions modify the semantics of a sentence, resulting in a new sentence that has a different meaning. Corruptions (12) check whether a metric can detect small lexical changes that cause the sentence's semantics to entirely change. Corruption (3) is designed to fool distributed and distributional representations of words, whose vectors often confuse synonyms and antonyms.

\subsection{Meaning-preserving corruptions}

Meaning-preserving corruptions change the lexical presentation of a sentence while still preserving meaning and fluency. For such transformations, the "corruption" is actually logically equivalent to the original sentence, and we would expect that consistent annotators would assign roughly the same score to each. These transformations include changes such as rephrasing a sentence from active voice to passive voice (4) or paraphrasing within a sentence (5).

\subsection{Fluency disruptions}

Beyond understanding semantics, metrics must also recognize when a sentence lacks fluency and grammar. Corruptions (7-9) were created for this reason, and do so by generating ungrammatical sentences. 


\begin{tabular}{|c|c|c|c|}
\hline \multicolumn{4}{|c|}{ Meaning Altering } \\
\hline 1 & negated subject (337) & "A man is playing a harp" & "There is no man playing a harp" \\
\hline 2 & negated action (202) & "A jet is flying" & "A jet is not flying" \\
\hline 3 & antonym replacement (246) & "a dog with short hair" & "a dog with long hair" \\
\hline \multicolumn{4}{|c|}{ Meaning Preserving } \\
\hline 4 & active-to-passive (238) & "A man is cutting a potato" & "A potato is being cut by a man" \\
\hline 5 & synonymous phrases $(240)$ & "A dog is eating a doll" & "A dog is biting a doll" \\
\hline 6 & determiner substitution (65) & "A cat is eating food" & "The cat is eating food" \\
\hline
\end{tabular}

Table 1: Corruptions from the SICK dataset. The left column lists the number of instances for each corruption type.

\begin{tabular}{|l|l|l|l|}
\hline \multicolumn{4}{|c|}{ Fluency disruptions } \\
\hline 7 & double PP (500) & "A boy walks at night" & "A boy walks at night at night" \\
8 & remove head from PP (500) & "A man danced in costume" & "A man danced costume" \\
9 & re-order chunked phrases (500) & "A woman is slicing garlics" & "Is slicing garlics a woman" \\
\hline
\end{tabular}

Table 2: Generated corruptions. The first column gives the total number of generated corruptions in parentheses.

\section{Metrics Overview}

\subsection{Existing Metrics}

Many existing metrics work by identifying lexical similarities, such as n-gram matches, between the candidate and reference sentences. Commonly-used metrics include BLEU, CIDEr, and TER:

- BLEU, an early MT metric, is a precisionbased metric that rewards candidates whose words can be found in the reference but penalizes short sentences and ones which overuse popular n-grams (Papineni et al., 2001).

- CIDEr, an image captioning metric, uses a consensus-based voting of tf-idf weighted ngrams to emphasize the most unique segments of a sentence in comparison (Vedantam et al., 2014).

- TER (Translation Edit Rate) counts the changes needed so the surface forms of the output and reference match (Snover et al., 2006).

Other metrics have attempted to capture similarity beyond surface-level pattern matching:

- METEOR, rather than strictly measuring ngram matches, accounts for soft similarities between sentences by computing synonym and paraphrase scores between sentence alignments (Denkowski and Lavie, 2014).
- BADGER takes into account the contexts over the entire set of reference sentences by using a simple compression distance calculation after performing a series of normalization steps (Parker, 2008).

- TERp (TER-plus) minimizes the edit distance by stem matches, synonym matches, and phrase substitutions before calculating the TER score, similar to BADGER's normalization step (Snover et al., 2009).

We evaluate the strengths and weaknesses of these existing metrics in Section 7.

\subsection{Toy Metric: W2V-AVG}

To demonstrate how this paper's techniques can also be applied to measure a new evaluation metric, we create a toy metric, W2V-AVG, using the cosine of the centroid of a sentence's word2vec embeddings (Mikolov et al., 2013). The goal for this true bagof-words metric is to serve as a sanity check for how corruption unit tests can identify metrics that capture soft word-level similarities, but cannot handle directed relationships between entities.

\section{Datasets}

\subsection{SICK}

All of our experiments are run on the Sentences Involving Compositional Knowledge (SICK) dataset, 
which contains entries consisting of a pair of sentences and a human-estimated semantic relatedness score to indicate the similarity of the sentence pair (Marelli et al., 2014). The reason we use this data is twofold:

1. it is a well-known and standard dataset within semantic textual similarity community.

2. it contains many common sentence transformation patterns, such as those described in Table 1 .

The SICK dataset was built from the 8K ImageFlickr dataset ${ }^{1}$ and the SemEval 2012 STS MSRVideo Description corpus ${ }^{2}$. Each of these original datasets contain human-generated descriptions of images/videos - a given video often has 20-50 reference sentences describing it. These reference sets prove very useful because they are more-or-less paraphrases of one another; they all describe the same thing. The creators of SICK selected sentence pairs and instructed human annotators to ensure that all sentences obeyed proper grammar. The creators of SICK ensured that two of the corruption types - meaning-altering and meaning-preserving - were generated in the annotated sentence pairs. We then filtered through SICK using simple rule-based templates $^{3}$ to identify each of the six corruption types listed in Table 1. Finally, we matched the sentences in the pair back to their original reference sets in the Flickr8 and MSR-Video Description corpora to obtain reference sentences for our evaluation metrics experiments.

\subsection{SICK+}

Since all of the entries in the SICK dataset were created for compositional semantics, every sentence was manually checked by annotators to ensure fluency. For our study, we also wanted to measure

\footnotetext{
${ }^{1}$ http://nlp.cs.illinois.edu/ HockenmaierGroup/data.html

${ }^{2}$ http: / / www.cs.york.ac.uk/ semeval-2012/task6/index.php?id=data

${ }^{3}$ For instance, the "Antonym Replacement" template checked to see if the two sentences were one word apart, and if so whether they had a SICK-annotated NOT_ENTAILMENT relationsip.
}

the effects of bad grammar between sentences, so we automatically generated our own corruptions to the SICK dataset to create SICK+, a set of fluency-disrupting corruptions. The rules to generate these corruptions were simple operations involving chunking and POS-tagging. Fortunately, these corruptions were, by design, meant to be ungrammatical, so there was no need for (difficult) automatic correctness checking for fluency.

\section{Inconsistencies in Human Judgment}

A major issue with comparing metrics against human judgment is that human judgments are often inconsistent. One reason for this is that high-level semantic tasks are difficult to pose to annotators. Consider SICK's semantic relatedness annotation as a case study for human judgment. Annotators were shown two sentences, were asked "To what extent are the two sentences expressing related meaning?", and were instructed to select an integer from 1 (completely unrelated) to 5 (very related). We can see the difficulty annotators faced when estimating semantic relatedness, especially because the task description was intentionally vague to avoid biasing annotator judgments with strict definitions. In the end, "the instructions described the task only through [a handful of] examples of relatedness" (Marelli et al., 2014).

As a result of this hands-off annotation guideline, the SICK dataset contains glaring inconsistencies in semantic relatedness scores, even for sentence pairs where the only difference between two sentences is due to the same known transformation. Figure 1 demonstrates the wide range of human-given scores for the pairs from SICK that were created with the Negated Subject transformation. Since the guidelines did not establish how to handle the effect of this transformation, some annotators rated it high for describing the same actions, while others rated it low for having completely opposite subjects.

To better appreciate the scope of these annotation discrepancies, Figure 2 displays the distribution of "gold" human scores for every instance of the "Negated Subject" transformation. We actually find that the relatedness score approximately follows a normal distribution centered at 3.6 with a standard 


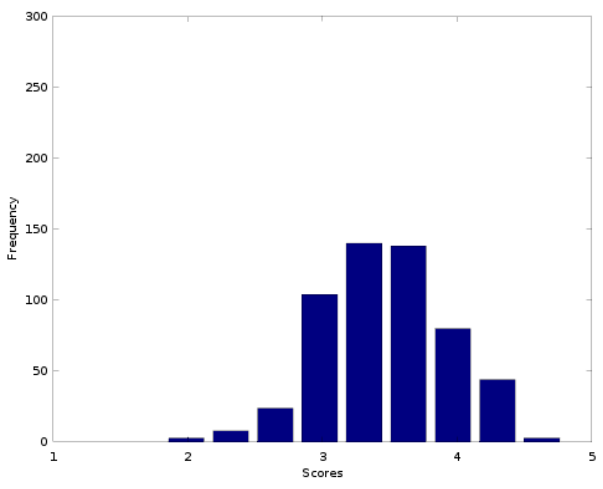

Figure 2: Human annotations for the Negated Subject corruption.

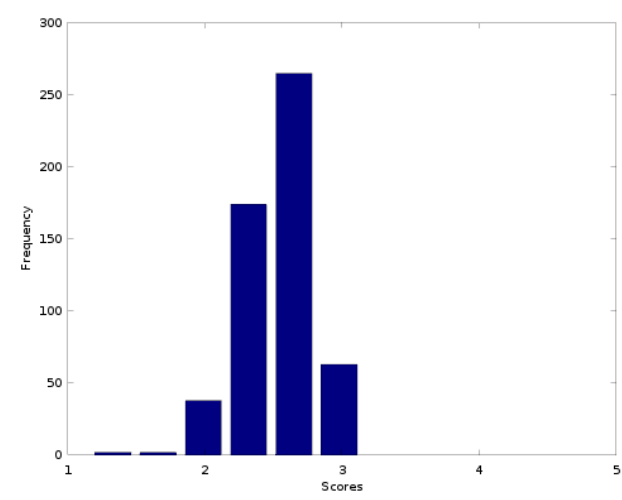

Figure 3: Metric predictions for the Negated Subject corruption.

deviation of about .45 . The issue with this distribution is that regardless of whether the annotators rank this specific transformation as low or high, they should be ranking it consistently. Instead, we see that their annotations span all the way from 2.5 to 4.5 with no reasonable justification as to why.

Further, a natural question to ask is whether all sentence pairs within this common Negated Subject transformation do, in fact, share a structure of how similar their relatedness scores "should" be. To answer this question, we computed the similarity between the sentences in an automated manner using three substantially different evaluation metrics: METEOR, BADGER, and TERp. These three metrics were chosen because they present three very different approaches for quantifying semantic similarity, namely: sentence alignments, compression redundancies, and edit rates. We felt that these different approaches for processing the sentence pairs would allow for different views of their underlying relatedness.

To better understand how similar an automatic metric would rate these sentence pairs, Figure 3 shows the distribution over scores predicted by the METEOR metric. The first observation is that the metric produces scores that are far more peaky than the gold scores in Figure 2, which indicates that they have a significantly more consistent structure about them.

In order to see how each metric's scores compare, Table 3 lists all pairwise correlations between the gold and the three metrics. As a sanity check, we can see that the $1.0 \mathrm{~s}$ along the diagonal indicate perfect correlation between a prediction and itself. More interestingly, we can see that the three metrics have alarmingly low correlations with the gold scores: $0.09,0.03$, and 0.07. However, we also see that the three metrics all have significantly higher correlations amongst one another: $0.80,0.80$, and 0.91. This is a very strong indication that the three metrics all have approximate agreement about how the various sentences should be scored, but this consensus is not at all reflected by the human judgments.

\section{MUTT Experiments}

In our Metric Unit TesTing experiments, we wanted to measure the fraction of times that a given metric is able to appropriately handle a particular corruption type. Each (original,corruption) pair is considered a trial, which the metric either gets correct or

\begin{tabular}{|l|l|l|l|l|}
\hline & gold & METEOR & BADGER & TERp \\
\hline gold & 1.00 & 0.09 & 0.03 & 0.07 \\
\hline METEOR & 0.09 & 1.00 & 0.91 & 0.80 \\
\hline BADGER & 0.03 & 0.91 & 1.00 & 0.80 \\
\hline TERp & 0.07 & 0.80 & 0.80 & 1.00 \\
\hline
\end{tabular}

Table 3: Pairwise correlation between the predictions of three evaluation metrics and the gold standard. 


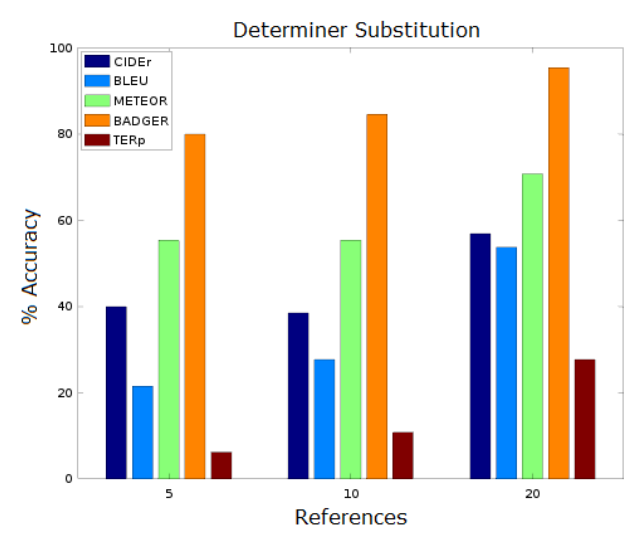

Figure 4: Results for the Determiner Substitution corruption (using Difference formula scores).

incorrect. We report the percent of successful trials for each metric in Tables 4, 5, and 6. Experiments were run using 5, 10, and 20 reference sentences to understand which metrics are able perform well without much data and also which metrics are able to effectively use more data to improve. An accuracy of $75 \%$ would indicate that the metric is able to assign appropriate scores 3 out of 4 times. ${ }^{4}$

For Meaning-altering and Fleuncy-disrupting corruptions, the corrupted sentence will be truly different from the original and reference sentences. A trial would be successful when the score of the original $s_{\text {orig }}$ is rated higher than the score of the corruption $s_{\text {corr }}$ :

$$
s_{\text {orig }}>s_{\text {corr }}
$$

Alternatively, Meaning-preserving transformations create a "corruption" sentence which is just as correct as the original. To reflect this, we consider a trial to be successful when the score of the corruption $s_{\text {corr }}$ is within $15 \%$ of the score of the original $s_{\text {orig }}$ :

$$
\left|\frac{s_{\text {orig }}-s_{\text {corr }}}{s_{\text {orig }}+\epsilon}\right| \leq 0.15
$$

where $\epsilon$ is a small constant $\left(10^{-9}\right)$ to prevent division by zero. We refer to this alternative trial formulation as the Difference formula.

\footnotetext{
${ }^{4}$ Our code is made available at https : / g ithub. com/text-machine-lab/MUTT
}

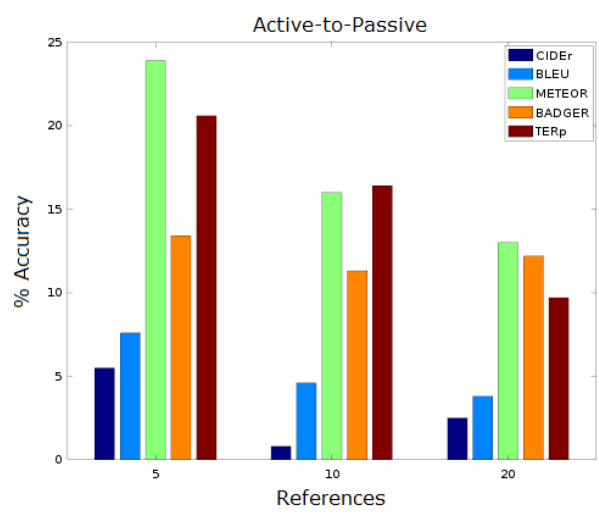

Figure 5: Results for the Active-to-Passive corruption (using Difference formula scores).

\section{Discussion}

\subsection{Meaning-altering corruptions}

As shown by the middle figure in Table 4 , it is CIDEr which performs the best for Antonym Replacement. Even with only a few reference sentences, it is already able to score significantly higher than the other metrics. We believe that a large contributing factor for this is CIDEr's use of tf-idf weights to emphasize the important aspects of each sentence, thus highlighting the modified when compared against the reference sentences.

The success of these metrics reiterates the earlier point about metrics being able to perform more consistently and reliably than human judgment.

\subsection{Meaning-preserving corruptions}

The graph in Figure 4 of the determiner substitution corruption shows an interesting trend: as the number of references increase, all of the metrics increase in accuracy. This corruption replaces " $a$ " in the candidate with a "the', or vice versa. As the references increase, there we tend to see more examples which use these determiners interchanagably while keeping the rest of the sentence's meaning the same. Since a large number of references results in far more for the pair to agree on, the two scores are very close.

Conversely, the decrease in accuracy in the 


\begin{tabular}{|l|l|l|l|}
\hline \multicolumn{4}{|c|}{ 1. Negated Subject } \\
\hline num refs & 5 & 10 & 20 \\
\hline CIDEr & 99.4 & 99.4 & 99.4 \\
\hline BLEU & 99.1 & 99.7 & 99.7 \\
\hline METEOR & 97.0 & 98.5 & 98.2 \\
\hline BADGER & 97.9 & 97.6 & 98.2 \\
\hline TERp & 99.7 & 99.7 & 99.4 \\
\hline \hline
\end{tabular}

\begin{tabular}{|l|l|l|l|}
\hline \multicolumn{4}{|c|}{ 2. Negated Action } \\
\hline num refs & 5 & 10 & 20 \\
\hline CIDEr & 98.5 & 98.5 & 98.5 \\
\hline BLEU & 97.5 & 97.5 & 98.0 \\
\hline METEOR & 96.0 & 96.0 & 97.0 \\
\hline BADGER & 93.6 & 95.5 & 96.5 \\
\hline TERp & 95.5 & 97.0 & 95.0 \\
\hline \hline
\end{tabular}

\begin{tabular}{|l|l|l|l|}
\hline \multicolumn{4}{|c|}{ 3. Antonym Replacement } \\
\hline num refs & 5 & 10 & 20 \\
\hline CIDEr & $\mathbf{8 6 . 2}$ & $\mathbf{9 2 . 7}$ & $\mathbf{9 3 . 5}$ \\
\hline BLEU & 76.4 & 85.4 & 88.6 \\
\hline METEOR & 80.9 & 86.6 & 91.5 \\
\hline BADGER & 76.0 & 85.8 & 88.6 \\
\hline TERp & 75.2 & 79.7 & 80.1 \\
\hline \hline
\end{tabular}

Table 4: Meaning-altering corruptions. These \% accuracies represent the number of times that a given metric was able to correctly score the original sentence higher than the corrupted sentence. Numbers referenced in the prose analysis are highlighted in bold.

\begin{tabular}{|l|l|l|l|}
\hline \multicolumn{4}{|c|}{ 4. Active-to-Passive } \\
\hline num refs & 5 & 10 & 20 \\
\hline CIDEr & 5.5 & 0.8 & 2.5 \\
\hline BLEU & 7.6 & 4.6 & 3.8 \\
\hline METEOR & $\mathbf{2 3 . 9}$ & $\mathbf{1 6 . 0}$ & $\mathbf{1 3 . 0}$ \\
\hline BADGER & 13.4 & 11.3 & 12.2 \\
\hline TERp & 20.6 & 16.4 & 9.7 \\
\hline \hline
\end{tabular}

\begin{tabular}{|l|l|l|l|}
\hline \multicolumn{4}{|c|}{ 5. Synonymous Phrases } \\
\hline num refs & 5 & 10 & 20 \\
\hline CIDEr & 32.1 & 26.2 & 30.0 \\
\hline BLEU & 45.0 & 36.7 & 34.2 \\
\hline METEOR & 62.1 & 62.1 & 62.1 \\
\hline BADGER & 80.8 & 80.4 & 86.7 \\
\hline TERp & 53.3 & 46.7 & 41.2 \\
\hline
\end{tabular}

\begin{tabular}{|l|l|l|l|}
\hline \multicolumn{4}{|c|}{ 6. DT Substitution } \\
\hline num refs & 5 & 10 & 20 \\
\hline CIDEr & 40.0 & 38.5 & 56.9 \\
\hline BLEU & 21.5 & 27.7 & 53.8 \\
\hline METEOR & 55.4 & 55.4 & 70.8 \\
\hline BADGER & $\mathbf{8 0 . 0}$ & $\mathbf{8 4 . 6}$ & $\mathbf{9 5 . 4}$ \\
\hline TERp & 6.2 & 10.8 & 27.7 \\
\hline \hline
\end{tabular}

Table 5: Meaning-preserving corruptions. These $\%$ accuracies represent the number of times that a given metric was able to correctly score the semantically-equaivalent "corrupted" sentence within $15 \%$ of the original sentence. Numbers referenced in the prose analysis are highlighted in bold.

\begin{tabular}{|l|l|l|l|}
\hline \multicolumn{4}{|c|}{ 7. Duplicate PP } \\
\hline num refs & 5 & 10 & 20 \\
\hline CIDEr & 100 & 99.0 & 100 \\
\hline BLEU & 100 & 100 & 100 \\
\hline METEOR & 95.1 & 98.5 & 99.5 \\
\hline BADGER & $\mathbf{6 3 . 5}$ & $\mathbf{7 0 . 0}$ & $\mathbf{7 4 . 9}$ \\
\hline TERp & 96.6 & 99.0 & 99.0 \\
\hline \hline
\end{tabular}

\begin{tabular}{|l|l|l|l|}
\hline \multicolumn{4}{|c|}{ 8. Remove Head From PP } \\
\hline num refs & 5 & 10 & 20 \\
\hline CIDEr & 69.5 & 76.8 & 80.8 \\
\hline BLEU & $\mathbf{6 3 . 5}$ & $\mathbf{8 1 . 3}$ & $\mathbf{8 7 . 7}$ \\
\hline METEOR & 60.6 & 72.9 & 84.2 \\
\hline BADGER & 63.1 & 67.0 & 71.4 \\
\hline TERp & 52.7 & 66.5 & 70.4 \\
\hline \hline
\end{tabular}

\begin{tabular}{|l|l|l|l|}
\hline \multicolumn{4}{|c|}{ 9. Re-order Chunks } \\
\hline num refs & 5 & 10 & 20 \\
\hline CIDEr & 91.4 & 95.6 & 96.6 \\
\hline BLEU & 83.0 & 91.4 & 94.2 \\
\hline METEOR & $\mathbf{8 1 . 2}$ & $\mathbf{8 9 . 6}$ & $\mathbf{9 2 . 4}$ \\
\hline BADGER & 95.4 & 96.6 & 97.8 \\
\hline TERp & 91.0 & 93.4 & 93.4 \\
\hline \hline
\end{tabular}

Table 6: Fluency-disrupting corruptions. These \% accuracies represent the number of times that a given metric was able to correctly score the original sentence higher than the corrupted sentence. Numbers referenced in the prose analysis are highlighted in bold.

Active-to-Passive table reflects how adding more (mostly active) references makes the system more (incorrectly) confident in choosing the active original. As the graph in Figure 5 shows, METEOR performed the best, likely due to its sentence alignment approach to computing scores.

\subsection{Fluency disruptions}

All of the metrics perform well at identifying the duplicate prepositional phrase corruption, except for BADGER which has noticeably lower accuracy scores than the rest. These lower scores may be attributed to the compression algorithm that it uses to compute similarity. Because BADGER's algorithm works by compressing the candidate and references jointly, we can see why a repeated phrase would be of little effort to encode - it is a compression algorithm, after all. The result of easy-to-compress redundancies is that the original sentence and its corruption have very similar scores, and BADGER gets fooled.

Unlike the other two fluency-disruptions, none of 
the accuracy scores of the "Remove Head from PP" corruption reach $90 \%$, so this corruption could be seen as one that metrics could use improvement on. BLEU performed the best on this task. This is likely due to its ngram-based approach, which is able to identify that deleting a word breaks the fluency of a sentence.

All of the metrics perform well on the "Re-order Chunks" corruption. METEOR, however, does slightly worse than the other metrics. We believe this to be due to its method of generating an alignment between the words in the candidate and reference sentences. This alignment is computed while minimizing the number of chunks of contiguous and identically ordered tokens in each sentence pair (Chen et al., 2015). Both the original sentence and the corruption contain the same chunks, so it makes sense that METEOR would have more trouble distinguishing between the two than the n-gram based approaches.

\subsection{W2V-AVG}

The results for the W2V-AVG metric's success on each corruption are shown in Table 7. "Shuffled Chunks" is one of the most interesting corruptions for this metric, because it achieves an accuracy of $0 \%$ across the board. The reason for this is that W2V-AVG is a pure bag-of-words model, meaning that word order is entirely ignored, and as a result the model cannot distinguish between the original sentence and its corruption, and so it can never rank the original greater than the corruption.

Surprisingly, we find that W2V-AVG is far less fooled by active-to-passive than most other metrics. Again, we believe that this can be attributed to its bag-of-words approach, which ignores the word order imposed by active and passive voices. Because each version of the sentence will contain nearly all of the same tokens (with the exception of a few "is" and "being" tokens), the two sentence representations are very similar. In a sense, W2V-AVG does well on passive sentences for the wrong reasons rather than understanding that the semantics are unchanged, it simply observes that most of the words are the same. However, we still see the trend that performance goes down as the number of reference

\begin{tabular}{|l|l|l|l|}
\hline \multicolumn{4}{|c|}{ average word2vec metric } \\
\hline num references & 5 & 10 & 20 \\
\hline 1. Negated Action & $\mathbf{7 2 . 8}$ & $\mathbf{7 4 . 5}$ & $\mathbf{6 0 . 7}$ \\
\hline 2. Antonym Replacement & 91.5 & 93.0 & 92.3 \\
\hline 3. Negated Subject & $\mathbf{9 8 . 2}$ & $\mathbf{9 9 . 1}$ & $\mathbf{9 7 . 8}$ \\
\hline 4. Active-to-Passive* & $\mathbf{8 4 . 9}$ & $\mathbf{8 3 . 6}$ & $\mathbf{8 0 . 3}$ \\
\hline 5. Synonymous Phrase* & 98.3 & 99.1 & 98.3 \\
\hline 6. DT Substitution* & 100.0 & 100.0 & 100.0 \\
\hline 7. Duplicate PP & 87.6 & 87.6 & 87.6 \\
\hline 8. Remove Head From PP & 78.4 & 82.5 & 82.5 \\
\hline 9. Shuffle Chunks & $\mathbf{0 0 . 0}$ & $\mathbf{0 0 . 0}$ & $\mathbf{0 0 . 0}$ \\
\hline \hline 1. Negated Action* & $\mathbf{1 0 0 . 0}$ & $\mathbf{1 0 0 . 0}$ & $\mathbf{1 0 0 . 0}$ \\
\hline 3. Negated Subject* & $\mathbf{8 2 . 8}$ & $\mathbf{8 7 . 3}$ & $\mathbf{8 7 . 1}$ \\
\hline \hline
\end{tabular}

Table 7: Performance of the AVG-W2V metric. These $\%$ accuracies represent the number of successful trials. Numbers referenced in the prose analysis are highlighted in bold. * indicates the scores computed with the Difference formula.

sentences increases.

Interestingly, we can see that although W2V-AVG achieved $98 \%$ accuracy on "Negated Subject", it scored only $75 \%$ on "Negated Action". This initially seems quite counter intuitive - either the model should be good at the insertion of a negation word, or it should be bad. The explanation for this reveals a bias in the data itself: in every instance where the "Negated Subject" corruption was applied, the sentence was transformed from "A/The [subject] is" to "There is no [subject]". This is differs from the change in "Negated Action", which is simply the insertion of "not" into the sentence before an action. Because one of these corruptions resulted in $3 \mathrm{x}$ more word replacements, the model is able to identify it fairly well.

To confirm this, we added two final entries to Table 7 where we applied the Difference formula to the "Negated Subject" and "Negated Action" corruptions to see the fraction of sentence pairs whose scores are within $15 \%$ of one another. We found that, indeed, the "Negated Action" corruption scored $100 \%$ (meaning that the corruption embeddings were very similar to the original embeddings), while the "Negated Subject" corruption pairs were only similar about $85 \%$ of the time. By analyzing these interpretable errors, we can see that stop 
words play a larger role than we'd want in our toy metric. To develop a stronger metric, we might change W2V-AVG so that it considers only the content words when computing the centroid embeddings.

\section{Conclusion}

The main contribution of this work is a novel approach for analyzing evaluation metrics for language generation tasks using Metric Unit TesTs. Not only is this evaluation procedure able to highlight particular metric weaknesses, it also demonstrates results which are far more consistent than correlation with human judgment; a good metric will be able to score well regardless of how noisy the human-derived scores are. Finally, we demonstrate the process of how this analysis can guide the development and strengthening of newly created metrics that are developed.

\section{References}

Chris Callison-Burch, Philipp Koehn, Christof Monz, Kay Peterson, Mark Przybocki, and Omar Zaidan. 2010. Findings of the 2010 joint workshop on statistical machine translation and metrics for machine translation. In Proceedings of the Joint Fifth Workshop on Statistical Machine Translation and MetricsMATR, pages 17-53, Uppsala, Sweden, July. Association for Computational Linguistics.

X. Chen, H. Fang, TY Lin, R. Vedantam, S. Gupta, P. Dollr, and C. L. Zitnick. 2015. Microsoft coco captions: Data collection and evaluation server. arXiv preprint arXiv:1504.00325.

Michael Denkowski and Alon Lavie. 2014. Meteor universal: Language specific translation evaluation for any target language. In Proceedings of the EACL 2014 Workshop on Statistical Machine Translation.

Chin-Yew Lin. 2004. Rouge: a package for automatic evaluation of summaries. pages 25-26.

Matous Machacek and Ondrej Bojar. 2014. Results of the wmt14 metrics shared task. In Proceedings of the Ninth Workshop on Statistical
Machine Translation, pages 293-301, Baltimore, Maryland, USA, June. Association for Computational Linguistics.

Nitin Madnani, Joel Tetreault, and Martin Chodorow. 2012. Re-examining machine translation metrics for paraphrase identification. In Proceedings of the 2012 Conference of the North American Chapter of the Association for Computational Linguistics: Human Language Technologies, pages 182-190, Montréal, Canada, June. Association for Computational Linguistics.

M. Marelli, S. Menini, M. Baroni, L. Bentivogli, R. Bernardi, R. Zamparelli, and Fondazione Bruno Kessler. 2014. A sick cure for the evaluation of compositional distributional semantic models.

Tomas Mikolov, Ilya Sutskever, Kai Chen, Greg Corrado, and Jeffrey Dean. 2013. Distributed representations of words and phrases and their compositionality. In In Proceedings of NIPS.

K. Papineni, S. Roukos, T. Ward, and W. Zhu. 2001. Bleu: a method for automatic evaluation of machine translation. Technical report, September.

Steven Parker. 2008. Badger: A new machine translation metric.

Matthew Snover, Bonnie Dorr, Richard Schwartz, Linnea Micciulla, and John Makhoul. 2006. A study of translation edit rate with targeted human annotation. In In Proceedings of Association for Machine Translation in the Americas, pages 223231.

Matthew G. Snover, Nitin Madnani, Bonnie Dorr, and Richard Schwartz. 2009. Ter-plus: Paraphrase, semantic, and alignment enhancements to translation edit rate.

Richard Socher, Eric H. Huang, Jeffrey Pennington, Andrew Y. Ng, and Christopher D. Manning. 2011. Dynamic Pooling and Unfolding Recursive Autoencoders for Paraphrase Detection. In Advances in Neural Information Processing Systems 24.

Ramakrishna Vedantam, C. Lawrence Zitnick, and Devi Parikh. 2014. Cider: Consensusbased image description evaluation. CoRR, abs/1411.5726. 